\title{
REFERENCES
}

1. E. T. Copson, Theory of functions of a complex variable, Oxford, 1935.

2. G. Doetsch, Theorie und Anwendung der Laplace Transformation, Berlin, Springer, 1937.

3. W. Magnus and F. Oberhettinger, Special functions of mathematical physics, New York, Chelsea, 1949.

4. D. V. Widder, The Laplace transform, Princeton, 1941.

University of Alberta

\section{ON FUNDAMENTAL MATRIX SOLUTIONS}

\section{HYMAN J. ZIMMERBERG}

In this note a recent result of Miller and Schiffer $[3, \S 3]$ will be extended to show how a fundamental matrix solution for the differential equations of a system of two point boundary value problems, written in matrix form as

$$
\mathcal{L}[y] \equiv y^{\prime}-A(x) y=0, \quad s[y] \equiv M y(a)+N y(b)=0,
$$

can be represented in terms of the Green's matrix of the system, or in terms of a special generalized Green's matrix in case the system (1) is compatible. For the system (1), $A(x)$ is an $n \times n$ matrix of complex-valued continuous functions of the real variable $x$ on the finite interval $a \leqq x \leqq b, M$ and $N$ are $n \times n$ complex constant matrices such that the rank of the $n \times 2 n$ matrix $\|M N\|$ is $n$, while the vector $y$ will be treated as an $n \times 1$ matrix.

1. In this section we shall assume that the system (1) is incompatible. Then a unique Green's matrix exists and is given by (Bliss $[1, \S 5])$

$$
G(x, t)=\frac{1}{2} Y(x)\left[\frac{|x-t|}{x-t} I+D^{-1} \Delta\right] Y^{-1}(t),
$$

where $Y(x)$ is a fundamental matrix solution of $\mathcal{L}[y]=0, D \equiv M Y(a)$ $+N Y(b), \Delta \equiv M Y(a)-N Y(b)$, and $I$ is the $n \times n$ identity matrix.

THEOREM. If $G(x, t)$ is the ordinary Green's matrix for an incompatible system (1) and $i_{1}, i_{2}, \cdots, i_{2 n}$ denotes a renumbering of the columns of the $n \times 2 n$ matrix $\|M N\|$ such that the $i_{1}, \cdots, i_{n}$ columns are linearly independent vectors, then the corresponding $i_{1}, \cdots, i_{n}$ columns of the $n \times 2 n$ matrix $\|G(x, a) G(x, b)\|$ may be chosen as the columns for a fundamental matrix solution of the differential equations $\mathcal{L}[y]=0$.

Presented, in part, to the Society, October 25, 1952, under the title On the Green's matrix of a differential system; received by the editors August 7, 1953. 
From (2) it is clear that each column of $\|G(x, a) G(x, b)\|$ is a solution of $\mathcal{L}[y]=0$, where it is understood that $G(a, a) \equiv G\left(a^{+}, a\right)$ $=\lim _{x \rightarrow a^{+}} G(x, a)$ and $G(b, b) \equiv G\left(b^{-}, b\right)=\lim _{x \rightarrow b}-G(x, b)$. Moreover, $n$ of these columns are linearly independent as one readily verifies directly from (2) that

$$
G(x, a) Y(a)-G(x, b) Y(b) \equiv Y(x) .
$$

The final result on the selection of the $n$ linearly independent columns then follows from the fact that

$$
s[\|G(x, a) G(x, b)\|]=\|M-N\|,
$$

which results from the well-known properties of the Green's matrix $M G(a, t)+N G(b, t)=0, G\left(t^{+}, t\right)-G\left(t^{-}, t\right)=I$ on $a<t<b$.

2. On the other hand, if the system (1) is compatible of index $r$, $0<r \leqq n$, Reid $[4, \S 3]$ has shown that principal generalized Green's matrices exist for this system. The first systematic study of generalized Green's functions was given by Elliott [2]. Of the family of principal generalized Green's matrices, one may be specialized by choosing particular matrices $\Psi(x)$ and $\Theta(x)$ in the definitions (27) and on page 454 of [4] so that each of the matrices (28) and (39) of $[4]$ is the identity matrix. In view of the simplification induced by such a choice of $\Psi(x)$ and $\Theta(x)$ made below, the associated principal generalized Green's matrix may be termed the Green's matrix for the compatible system (1). In this section we shall show how a fundamental matrix solution for $\mathcal{L}[y]=0$ can be represented in terms of this unique Green's matrix for the compatible system (1). The notation of Reid [4] will be employed.

Let $Y(x) \equiv\left\|r Y_{1}(x) \cdots{ }^{r} Y_{r}(x) Y_{r+1}(x) \cdots Y_{n}(x)\right\|$ be a fundamental matrix solution of $\mathcal{L}[y]=0$, where the first $r$ columns are $r$ linearly independent vector solutions of the system (1). As $Z(x) \equiv Y^{-1}(x)$ will then be a fundamental matrix solution of the adjoint differential equations (4) of [4], let $\Gamma$ be an $n \times n$ nonsingular constant matrix such that the first $r$ rows of $\Gamma Z(x)$ will also satisfy the adjoint boundary conditions (5) of [4]. Thus we may choose ${ }^{r} Z(x)$ as

$$
r Z(x) \equiv\left\|\begin{array}{ll}
I_{r} & 0 \\
0 & 0
\end{array}\right\| \Gamma Z(x)
$$

where $I_{r}$ denotes the $r \times r$ identity matrix. Then the first $r$ columns of $[1 /(b-a)] Y(x) \Gamma^{-1}$ may be chosen for the first $r$ columns of $\Psi(x)$, and the first $r$ rows of $[1 /(b-a)] Z(x)$ may be chosen for the first $r$ rows of $\Theta(x)$. 
Now, if $G(x, t)$ is the given Green's matrix for the compatible system (1) with the above choice of $\Psi(x)$ and $\Theta(x)$, it follows from relation (40) and Theorem 5 of [4] that $K(x, t) \equiv(\partial / \partial t) G(x, t)$ $+G(x, t) A(t)$ on $a \leqq t<x, x<t \leqq b$ is equal to $[1 /(b-a)]^{r} Y(x) Z(t)$. In particular, the maximum number of linearly independent columns of $K(x, a)$ on $a \leqq x \leqq b$ may be chosen as a suitable maximal set of $r$ linearly independent vector solutions of the compatible system (1). It is to be noted that when $K(x, t) \equiv 0, G(x, t)$ is an ordinary Green's matrix.

In addition, from Theorem 2 of [4] and relation (3) above we have that

$$
G(x, a) Y(a)-G(x, b) Y(b)=Y(x)+{ }^{r} Y(x) C,
$$

where, in Theorem 2, $G_{1}(x, t)$ is chosen as the function (16) of [4] and $C \equiv U(a) Y(a)-U(b) Y(b)$. Moreover, as $\int_{a}^{b} \Theta(x) G(x, t) d x=0$ from relation (37) of $[4]$,

$$
\begin{aligned}
0 & =\int_{a}^{b} \Theta(x)[G(x, a) Y(a)-G(x, b) Y(b)] d x \\
& =[1 /(b-a)] \int_{a}^{b}\left\|\begin{array}{cc}
I_{r} & 0 \\
0 & 0
\end{array}\right\| Z(x)\left[Y(x)+{ }^{r} Y(x) C\right] d x \\
& =\left\|\begin{array}{cc}
I_{r} & 0 \\
0 & 0
\end{array}\right\|+\left\|\begin{array}{cc}
I_{r} & 0 \\
0 & 0
\end{array}\right\| C ;
\end{aligned}
$$

and thus,

$$
{ }^{r} Y(x) C={ }^{r} Y(x)\left\|\begin{array}{ll}
I_{r} & 0 \\
0 & 0
\end{array}\right\| C=-{ }^{r} Y(x)\left\|\begin{array}{cc}
I_{r} & 0 \\
0 & 0
\end{array}\right\|=-{ }^{r} Y(x) .
$$

Consequently,

$$
G(x, a) Y(a)-G(x, b) Y(b)=\left\|0 \cdots 0 \quad Y_{r+1}(x) \cdots Y_{n}(x)\right\| .
$$

Form next $W(x, t) \equiv G(x, t)-x N(x, t)$, where $N(x, t) \equiv(\partial / \partial x) G(x, t)$ $-A(x) G(x, t)$ on $a \leqq x<t, t<x \leqq b$. As $N(x, t)=-[1 /(b-a)]$ - $Y(x) \Gamma^{-1}{ }^{r} Z(t)$ from Theorem 3 of [4], one readily verifies that

$$
(\partial / \partial x) W(x, t)-A(x) W(x, t) \equiv 0 \text { on } a \leqq x<t, t<x \leqq b,
$$

and that

$$
W(x, a) Y(a)-W(x, b) Y(b)=\left\|0 \cdots 0 Y_{r+1}(x) \cdots Y_{n}(x)\right\| .
$$

Thus each column of $\|W(x, a) W(x, b)\|$, where $W(a, a) \equiv W\left(a^{+}, a\right)$ and 
$W(b, b) \equiv W\left(b^{-}, b\right)$, is a vector solution of $\mathcal{L}[y]=0$; and, moreover, $n-r$ of these columns are linearly independent solutions of the differential equations of (1) which do not satisfy the boundary conditions $s[y]=0$.

Therefore, if $j_{1}, \cdots, j_{n \rightarrow r}$ denotes $n-r$ columns of the $n \times 2 n$ matrix $s[\|W(x, a) W(x, b)\|]$ which are linearly independent, then the corresponding $j_{1}, \cdots, j_{n-r}$ columns of $\|W(x, a) W(x, b)\|$ together with the $r$ columns obtained above from $K(x, a)$ will form a suitable fundamental matrix solution for $\mathcal{L}[y]=0$.

\section{BIBLIOGRAPHY}

1. G. A. Bliss, $A$ boundary value problem for a system of ordinary linear differential equations of the first order, Trans. Amer. Math. Soc. vol. 28 (1926) pp. 561-584.

2. W. W. Elliott, Generalized Green's functions for compatible differential systems, Amer. J. Math. vol. 50 (1928) pp. 243-258.

3. K. S. Miller and M. M. Schiffer, On the Green's functions of ordinary differential systems, Proc. Amer. Math. Soc. vol. 3 (1952) pp. 433-441.

4. W. T. Reid, Generalized Green's matrices for compatible systems of differential equations, Amer. J. Math. vol. 53 (1931) pp. 443-459.

RUTGERS UNIVERSITY 